Braz J Med Biol Res, August 2012, Volume 45(8) 737-745

doi: 10.1590/S0100-879X2012007500085

Prostate-specific membrane antigen can promote in vivo osseous metastasis of prostate cancer cells in mice

Liang-Yun Zhao, Xiao-Peng Mao, Kai-Yuan Chao, Sheng-Jie Guo and Shao-Peng Qiu

The Brazilian Journal of Medical and Biological Research is partially financed by

\section{Q}

Ministério da Ciência e Tecnologia

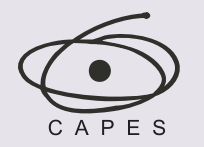

Ministério da Educação
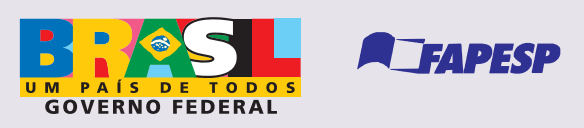

Institutional Sponsors

Ф SHIMADZU UNICAMP

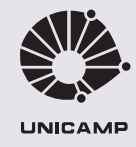

M PAIS DETTODO
GOVERNO FEDERAL
FAPESP

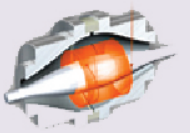

1. Associação Explore High - Performance MS Orbitrap Technology In Proteomics \& Metabolomics \section{analitica Thermo}

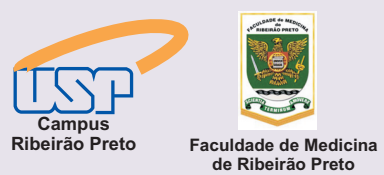

de Ribeirão Preto

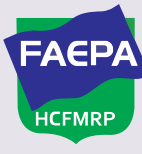




\title{
Prostate-specific membrane antigen can promote in vivo osseous metastasis of prostate cancer cells in mice
}

\author{
Liang-Yun Zhao ${ }^{1 *}$, Xiao-Peng Mao ${ }^{2 *}$, Kai-Yuan $\mathrm{Chao}^{3}$, \\ Sheng-Jie Guo ${ }^{2}$ and Shao-Peng Qiu ${ }^{2}$ \\ ${ }^{1}$ Department of Urology, Huangpu Hospital, The First Affiliated Hospital, Sun Yat-Sen University, \\ Guangzhou, Guangdong, China \\ ${ }^{2}$ Department of Urology, The First Affiliated Hospital, Sun Yat-Sen University, \\ Guangzhou, Guangdong, China \\ ${ }^{3}$ Research Centre for Clinical Laboratory Standard, Zhongshan School of Medicine, \\ Sun Yat-Sen University, Guangzhou, Guangdong, China
}

\begin{abstract}
Reports remain insufficient on whether and how prostate-specific membrane antigen (PSMA) can influence in vivo osseous metastasis of prostate cancer (PCa). In the present study, the authors induced stable expression of PSMA in mouse PCa cell line RM-1. In vivo osseous metastasis was induced in 37 6-week-old female C57BL/6 mice weighing $22.45 \pm 0.456 \mathrm{~g}$. RM-1 cells were actively injected into the femoral bone cavity, leading to bilateral dissymmetry of bone density in the femoral bone. Tumor cells were also detected in bone tissue by pathological examination. The impact on bone density was demonstrated by the significant difference between animals injected with RM-PSMA cells $\left(0.0738 \pm 0.0185 \mathrm{~g} / \mathrm{cm}^{2}\right)$ and animals injected with RM-empty plasmid cells $\left(0.0895 \pm 0.0241 \mathrm{~g} / \mathrm{cm}^{2}\right)$. The lytic bone lesion of the RM-PSMA group $(68.4 \%)$ was higher than that of the control group (27.8\%). Immunohistochemistry showed that the expression of both vascular endothelial growth factor (VEGF) and matrix metalloproteinase-9 (MMP-9) was distinctly higher in the RM-PSMA group than in the control group, while ELISA and Western blot assay indicated that VEGF and MMP-9 were higher in the RM-PSMA group compared to the control group (in vitro). Thus, the present study proposed and then confirmed for the first time that PSMA can promote in vivo osseous metastasis of PCa by increasing sclerotic destruction of PCa cells. Further analyses also suggested that PSMA functions positively on the invasive ability of RM-1 by increasing the expression of MMP-9 and VEGF by osseous metastases in vivo.
\end{abstract}

Key words: Prostate carcinoma; Prostate-specific membrane antigen; In vitro; Osseous metastasis

\section{Introduction}

Prostate cancer (PCa) is among the most frequently diagnosed cancers of the genitourinary system, representing the second most frequent cause of male malignant tumors (1). Prostate-specific membrane antigen (PSMA), an $\alpha$-transmembrane glycoprotein, has a noticeably higher expression in prostate carcinoma tissue than in normal and hyperplastic prostate epithelial tissue $(2,3)$ and its expression in androgen-independent and metastatic PCa tissues is further enhanced. This suggests that PSMA is probably intimately correlated with the degree of malignancy and metastasis of $\mathrm{PCa}$ (4). Thus, it is not surprising that PSMA, with its high specificity for the prostate, has attracted a great deal of attention as a target for PCa diagnosis and therapy.

The measurement of serum PSMA has been extensively studied (5). Some studies have focused on immunotherapy of PSMA based on the use of combined vaccines, vaccines with dendritic cells, and on tumor immunity and autoimmunity $(6,7)$, etc. Some studies have used radioimmunoscintigraphy of PSMA to detect PCa recurrence and metastasis (8). All of these studies have achieved some curative effects, but the exact mechanism of action and the effects of PSMA involved in carcinogenesis and PCa tumor progression still need further, more adequate exploration.

Correspondence: Shao-Peng Qiu and/or Xiao-Peng Mao, Department of Urology, The First Affiliated Hospital, Sun Yat-Sen University, Guangzhou, Guangdong 510080, China. E-mail: qiushp@mail.sysu.edu.cn and/or mxpzc1979@yahoo.com.cn

*These authors contributed equally to this study.

Received April 28, 2011. Accepted April 12, 2012. Available online May 18, 2012. Published August 3, 2012. 
The high rate of osseous metastases represents the major mutilation and cause of death due to PCa $(9,10)$. Recent investigations have proposed that PSMA plays a decisive part in the growth, invasion and migration of prostate cancer cell lines (11). Barwe et al. (12) reported that PSMA could promote the in vitro adhesion of $\mathrm{PCa}$ cells to bone marrow matrix and proposed that PSMA may play an important role in the occurrence of osseous metastases in PCa. However, no significant experiment has yet been reported demonstrating adequately the function of PSMA.

Some relevant studies have probed into the overexpression of vascular endothelial growth factor (VEGF) in $\mathrm{PCa}$ cell lines of primary and metastatic carcinoma tissue of $\mathrm{PCa}$ patients, leading to the reasonable deduction that VEGF could play an important role in the osseous metastasis of $\mathrm{PCa}(11,13)$. It was also reported that PSMA could promote tumor angiogenesis by increasing the invasion and migration ability of blood vessel endothelial cells $(14,15)$. Since degradation of the basement membrane and extracellular matrix by matrix metalloproteinase (MMPs) secreted by invasive tumors is the decisive factor for carcinoma infiltration and metastasis, some investigators presume that PSMA probably functions by regulating the invasiveness of PCa through its regulatory effect on MMP secretion $(16,17)$. Regretfully, further detailed investigations of the direct correlations between PSMA, VEGF and MMPs are scarce.

Based on these considerations and assumptions, the objective of the present study was to construct animal models of in vivo PCa osseous metastasis and, in subsequent studies, to deal with the in vivo regulation of $\mathrm{PCa}$ osseous metastases by PSMA in order to determine whether and how PSMA influences the osseous metastases of PCa in vivo. Using the proposed experiments and tests, we hope to attain new targets for the prevention, diagnosis and therapy of PCa osseous metastasis.

\section{Material and Methods}

\section{Materials \\ Eukaryotic expression plasmid pcDNA3.0/PSMA, pcDNA3.0/empty plasmid, the human PCa cell line LNCaP, and the mouse PCa cell line RM-1 were preserved in our laboratory (obtained from the American Type Culture Collection, USA). C57BL/6 female mice (6 weeks) were}

supplied by the Animal Experiment Center of Sun Yat-Sen University (China).

\section{Transfection of PSMA into RM-1 cells}

PSMA cDNA was subcloned into the pcDNA3.0 vector. As reported previously (18), the constructed plasmid DNA was purified from transformed $\mathrm{DH} 5-\alpha$ bacteria and stably transfected into RM-1 cells using the Lipofectin 2000 reagent (Invitrogen, Life Technologies, USA). Cells were selected with the antibiotic $\mathrm{G} 418$ (400 mg/mL) until discrete colonies appeared. Colonies were isolated and expanded for detection of PSMA expression. Under controlled conditions, RM-1 cells were transfected with the pcDNA3.0 plasmid alone. RM-1 cells not transfected with any plasmid were kept and used as control.

\section{RNA extraction and RT-PCR}

RNA was isolated from cells, and PSMA mRNA expression was detected by RT-PCR. In brief, total RNA was isolated from all cell lines (RM-1, LNCaP) using TRIzol Reagent (Invitrogen, USA) according to manufacturer instructions. Total RNA was mixed with the oligo(dT) 20 primer and incubated at $65^{\circ} \mathrm{C}$ for $5 \mathrm{~min}$. cDNA was then synthesized using Superscript III (Invitrogen) at $50^{\circ} \mathrm{C}$ for $50 \mathrm{~min}$, followed by heating at $85^{\circ} \mathrm{C}$ for $5 \mathrm{~min}$.

PSMA cDNA was synthesized and cloned from RM-1 and LNCaP cell lines by RT-PCR. The primers and conditions for PCR [PSMA and m-glyceraldehyde 3-phosphate dehydrogenase (mGAPDH)] are indicated in Table 1. In brief, the forward PSMA primer was 5'-TCTGCTCGCGCCGAG ATGTG-3', the reverse primer was 5'-ATTTTATAAACCACC CGAAG-3', and the product size was about $148 \mathrm{bp}$. The forward primer of mGAPDH was 5'-CCCATGGCAAATTCC ATGGCACCG-3', the reverse primer was 5'-GTCATGG ATGACCTTGGCCAGGGG-3', and the product size was about 344 bp. PCR amplification was carried out with the platinum Taq DNA polymerase high fidelity (Invitrogen) in a total reaction volume of $25 \mu \mathrm{L}$ containing $2 \mu \mathrm{L}$ synthesized cDNA, $2 \mu \mathrm{L}$ each of the specific primer pair, $2.5 \mu \mathrm{L}$ 10X Taq buffer, $17.5 \mu \mathrm{L}$ sterilized water, and $1 \mu \mathrm{L}$ dNTP. A total of 35 cycles was completed with the following profile: cycle $1,94^{\circ} \mathrm{C}$ for $3 \mathrm{~min}$; cycles 2 through $34,94^{\circ} \mathrm{C}$ for 30 $\mathrm{s}, 55^{\circ} \mathrm{C}$ for $30 \mathrm{~s}$, and $72^{\circ} \mathrm{C}$ for $2 \mathrm{~min}$. The final reaction was extended for $10 \mathrm{~min}$ at $72^{\circ} \mathrm{C}$. The PCR products for PSMA and mGAPDH were analyzed by $1.5 \%$ agarose gel

Table 1. Primers and conditions for PCR.

\begin{tabular}{lllcc}
\hline $\begin{array}{l}\text { Objective } \\
\text { gene }\end{array}$ & Forward primer (5'-3') & Reverse primer (5'-3') & $\begin{array}{c}\text { Annealing } \\
\text { temperature }\left({ }^{\circ} \mathrm{C}\right) \text { ) }\end{array}$ & $\begin{array}{c}\text { Size of PCR } \\
\text { products (bp) }\end{array}$ \\
\hline PSMA & TCTGCTCGCGCCGAGATGTG & ATTTTATAAACCACCCGAAG & 55 & 148 \\
mGAPDH & CCC ATG GCAAAT TCC ATG GCA CCG & GTC ATG GAT GAC CTT GGC CAG GGG & 58 & 344 \\
\hline
\end{tabular}

PSMA = prostate-specific membrane antigen; $m G A P D H=$-glyceraldehyde 3-phosphate dehydrogenase . 
electrophoresis in TAE buffer and visualized by ethidium bromide staining.

\section{Western blot detection of PSMA expression}

For Western blot analyses, crude cell lysates were isolated from cells with ice-cold radioimmunoprecipitation assay (RIPA) lysis buffer. In brief, the protein concentrations were estimated by the bicinchoninic acid (BCA) assay (BCA assay kit, Nanjiing KeyGen, China) with ultraviolet spectrophotometry at $260 \mathrm{~nm}$ wavelength. About $40 \mu \mathrm{g}$ protein was run on a $10 \%$ SDS-polyacrylamide gel and blotted onto a polyvinylidene difluoride (PVDF) membrane. After blocking for $3 \mathrm{~h}$ with $5 \%$ skim milk in Tris-buffered saline Tween (TBST), PSMA (YPSMA-1, Abcam, USA) antibody (1:1000 dilution) and $\beta$-actin antibody (1:1000 dilution) were applied to PVDF membranes, followed by overnight incubation at $4^{\circ} \mathrm{C}$. The membranes were then washed with TBST and treated with horseradish peroxidase-conjugated goat anti-mouse IgG antibody (1:2000 dilution). After washing with TBST again, the membrane was developed with ECL Western blotting detection reagents (Pierce, USA).

\section{In vitro quantification of VEGF protein by enzyme-linked immunosorbent assay (ELISA)}

RM-1, RM-1 transfected with PSMA, and RM-1 transfected with empty plasmid cells $\left(2 \times 10^{5}\right.$ cells/well each) were plated onto six-well plates in RPMI-1640 containing $5 \%$ FCS. The cells were allowed to grow for $48 \mathrm{~h}$ until they were about $60-70 \%$ confluent. The growth medium was then removed and replaced with fresh RPMI-1640 containing $1 \%$ FCS. The cells were incubated for a further $24 \mathrm{~h}$ until about $80 \%$ confluence was attained. The medium was then harvested and filtered for the measurement of secretory VEGF. The remaining cells were collected and the viable cells were counted. VEGF present in the growth medium was measured using a Quantakine Human VEGF ELISA kit according to manufacturer instructions (R\&D Systems, USA). The concentration of VEGF was measured as picograms per milliliter $(\mathrm{pg} / \mathrm{mL})$ in the growth medium and the results were then calculated as picograms of secreted VEGF per cell. Each experiment was performed three times and the mean concentration of VEGF secretion was presented as the final result. The standard deviation (SD) of the means was used as error bars.

Western blot detection of MMP-9 expression (in vitro)

For Western blot analyses, crude cell lysates were isolated from cells using ice-cold RIPA lysis buffer. In brief, protein concentrations were estimated by BCA assay with ultraviolet spectrophotometry at $260 \mathrm{~nm}$ wavelength. About $40 \mu \mathrm{g}$ protein was run on a $10 \%$ SDS-polyacrylamide gel and blotted onto a PVDF membrane. After blocking for $3 \mathrm{~h}$ with 5\% skim milk in TBST, goat anti-mouse MMP-9 (Beijing Biosynthesis, China) antibody (1:200 dilution) or $\beta$-actin antibody (1:1000 dilution) was applied to PVDF membranes, followed by overnight incubation at $4^{\circ} \mathrm{C}$. The membranes were then washed with TBST and treated with horseradish peroxidase-conjugated mouse anti-goat IgG antibody (1:2000 dilution). After washing with TBST again, the membrane was developed with the ECL Western blotting detection reagents (Pierce).

\section{Construction of an animal model of PCa osseous metastasis and determination of bone densitometry}

Ten mice were allocated to each cell line and the experiment was repeated twice. In brief, RM-1 cells (transfected with PSMA or with empty plasmid) were plated and cultured to the exponential growth phase, then trypsinized, enumerated, and resuspended to a final concentration of $25 \times 10^{5}$ cells/mL in RPMI- 1640 containing $1 \%$ FBS and $10 \mathrm{~g} / \mathrm{LBSA}$. Suspensions of RM-1 cells $(20 \mu \mathrm{L})$ were injected by insertion of a 27-gauge needle through the mouse skin directly into the cavity of the right femoral bone of C57BL/6 mice (6 weeks old) anesthetized with $3 \%$ carbrital (Sigma, USA) based on weight $(60 \mathrm{mg} / \mathrm{kg})$. RPMI-1640 medium $(20 \mu \mathrm{L})$ was injected into the cavity of the left femoral bone for control. When dissymmetry of bilateral lower limb action was observed, bone density was determined under anesthesia using the Discovery A S/N 82239 bone density tester (Hologic, USA). If osseous metastasis was confirmed, the animals were dissected for further pathologic examination.

\section{Pathologic and immunohistochemical examination}

In this phase, tissue sections were taken from both femoral bones of the dissected animals. Once identified, 4- $\mu \mathrm{m}$ sections were cut from the appropriate blocks and mounted on slides for evaluation. In brief, 4- $\mu$ m paraffinembedded tissue sections were deparaffinized in xylene following de-xylene, stained with $\mathrm{HE}$ and mounted on slides with resin for evaluation.

Immunohistochemistry was performed by indirect immunoperoxidase staining as described in our previous reports (19-21). In brief, immunoglobulin G (lgG) was applied as a control slide to a matched set of slides. The 4- $\mu \mathrm{m}$ paraffinembedded tumor sections were deparaffinized in xylene, rehydrated in graded alcohol $(100,95,70$, and $50 \%)$, and immersed in methyl alcohol containing $0.01 \% \mathrm{H}_{2} \mathrm{O}_{2}$ for 10 $\min$. Sections were allowed to air dry and were then blocked with $10 \%$ normal goat serum in PBS, $\mathrm{pH} 7.4$, for VEGF and MMP-9 determination at room temperature (RT) for $1 \mathrm{~h}$.

The sections were then washed three times in PBS. Goat anti-mouse VEGF or goat anti-mouse MMP-9 in 5\% blocking serum (1:100) was added, followed by overnight incubation at $4^{\circ} \mathrm{C}$. After the incubation period, sections were kept at $37^{\circ} \mathrm{C}$ for $20 \mathrm{~min}$ before being washed three times in PBS. Biotinylated anti-goat/rabbit lgG antibody (GK500705, Gene Tech, China) was added to the sections followed by incubation for 30 min at RT. Sections were then stained with Dolichos biflorus agglutinin (DBA) solution for 5 min after being washed three times again in PBS. Distilled water was 
then used to wash the sections, which were counterstained with hematoxylin for $5 \mathrm{~min}$. Next, the slides were washed in distilled water, dipped in dilute ammonium hydroxide, washed in distilled water again, and finally mounted in crystal mounting solution for microscopic evaluation.

The extent of positivity was determined by immunohistochemical staining as follows: brown-yellow, light brown or buff staining of the cell membrane and cytoplasm indicated positive expression of VEGF (or MMP-9). VEGF (or MMP-9) expression was identified by the summation of stain intensity and positive cell rates. According to 5 randomly observed fields magnified 400X on each slide, the expression of VEGF (or MMP-9) was defined as negative ( $\leq 5 \%$ positive cells), weakly positive (5-25\% positive cells), and strongly positive ( $\geq 25 \%$ positive cells).

\section{Statistical analyses}

All statistical analyses were performed using the SPSS 11.5 software (SPSS, Inc., USA) and Microsoft Excel 2003. The results were analyzed by two- or three-way analysis of variance or the Student $t$-test. The differences in expression VEGF between PSMA-transfected RM-1 cells, negative control cells and RM-1 cells were determined by one-way ANOVA. The level of significance was set at $P<0.05$ in all analyses.

\section{Results}

PSMA mRNA transcripts in cell lines

In Figure 1A, RT-PCR demonstrates that the transcripts of PSMAcDNAwere detected in LNCaP (the positive control) and PSMA-transfected RM-1 cells, whose size appears to be in good agreement with the expected $148 \mathrm{bp}$, but not in negative control cell lines. The 344-bp RT-PCR product bands, size of mGAPDH cDNA, were detected in all cell lines (lanes 3-6 marked at $344 \mathrm{bp}$ ).

\section{Stable expression of the PSMA protein in transfected RM-1 cells}

The YPSMA-1 monoclonal antibody (mAb) is used to detect PSMA both in immunofluorescence and Western blot experiments. In Western blot assay, as shown in Figure 1B, PSMA protein (MW $100 \mathrm{kDa}$, as expected) was detected in PSMA-transfected RM-1 cells (Figure 1B, lane 1) and LNCaP cells (Figure 1B, lane 4 , the positive control), but not in negative control cell lines. The $\beta$-actin protein (MW $43 \mathrm{kDa}$ ) was detected in all cell lines.

\section{In vitro quantification of the VEGF protein in PCa cells}

As shown in Figure 2A, using ELISA we also found that the stable transfectants (PSMA) obviously promoted VEGF protein secretion $(25.4 \pm 1.2 \mathrm{pg} /$ cell $)$, compared to RM- 1 cells (15.6 $\pm 0.45 \mathrm{pg} /$ cell) and negative control cells (15.2 $\pm 0.96 \mathrm{pg} / \mathrm{cell})$, with a significant difference by oneway ANOVA ( $f=122.16, P<0.01$ ). These results further indicate the association between PSMAand VEGF secretion in prostate cancer cells.

\section{In vitro expression of the MMP-9 protein in PCa cells}

In Figure 2B, Western blot assay revealed that MMP-9 protein (MW $78 \mathrm{kDa}$ ) was detected in PSMA-transfected RM-1 cells (Figure 2B, lane 1), negative control cells (Figure $2 \mathrm{~B}$, lane 2 ) and $\mathrm{RM}-1$ cells (Figure $2 \mathrm{~B}$, lane 3 ). The expression of MMP-9 in PSMA-transfected RM-1 cells was higher than in negative control cells. The $\beta$-actin protein (MW 43 $\mathrm{kDa}$ ) were detected in all cell lines.

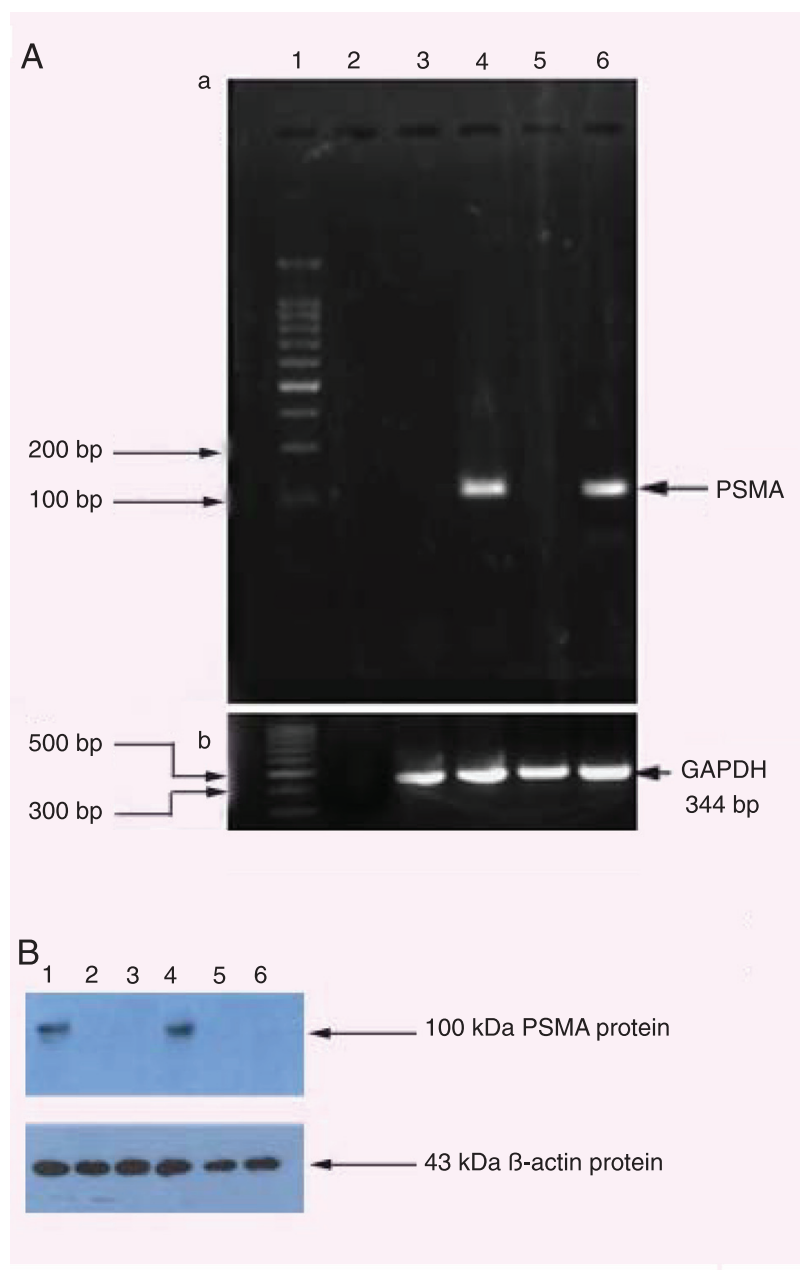

Figure 1. PSMA mRNA transcripts by RT-PCR and PSMA/ $\beta$-actin expression by Western blot. A, Lane $1=100$-bp DNA marker; lane 2 = negative control; lane $3=\mathrm{cDNA}$ in RM-1 cells; lane $4=$ cDNA in LNCaP cells; lane $5=$ cDNA in RM-empty plasmid; lane $6=$ cDNA in RM-PSMA cells. $a$, PSMA mRNA transcripts were detected only in RM-PSMA and LNCaP cells, but not in other carcinoma cells. $b$, mGAPDH cDNA was detected in each cell. $B$, Lane 1 = RM-PSMA protein; lanes 2 and $5=\mathrm{RM}-1$ protein; lanes 3 and $6=$ RM-empty plasmid protein; lane $4=\mathrm{LNCaP}$ protein. PSMA = prostate-specific membrane antigen; GADPH = glyceraldehyde 3-phosphate dehydrogenase. 


\section{Osseous metastasis modeling and bone densitometry detection}

Ten mice were allocated to each cell line and the experiment was repeated twice. Two weeks after the injection of RM-1 cell lines into the mouse femoral bone cavity, dissymmetry of bilateral lower limb action was observed in all the 37 surviving mice (three mice, 2 injected with empty-plasmid-transfected RM-1 cells and 1 injected with PSMA-transfected RM-1 cells, died of extreme anesthesia). Differences in bone density between the two femoral bones were then confirmed under anesthesia (Figure 3). Thus, the animal model of osseous metastasis employed in the present study proved to be successful.

Furthermore, as shown in Table 2, the osteolytic lesions of the PSMA-transfected RM-1 group were distinctly greater than those of the empty-plasmid-transfected RM-1 group (68.4 to $27.8 \%$ ), with a statistically significant difference shown by the chi-square test $\left(X^{2}=6.112, P=0.013, P<0.05\right)$.

The bone density differed significantly between animals injected with PSMA-transfected RM-1 cells $(0.0738 \pm 0.0185$ $\mathrm{g} / \mathrm{cm}^{2}$ ) and animals injected with empty-plasmid-transfected $\mathrm{RM}-1$ cells $\left(0.0895 \pm 0.0241 \mathrm{~g} / \mathrm{cm}^{2}\right)$, as determined by the $t$-test $(t=2.237, \mathrm{P}=0.032, \mathrm{P}<0.05)$. Pathologic examination of all animals injected with RM-1 PCa cells revealed that numerous tumor cells were destroying bone tissue inside the femoral bone cavity.

\section{Infection with PSMA promotes the expression of VEGF on PCa osseous metastasis tissue}

Nineteen mice injected with PSMAtransfected RM-1 cells and 18 mice injected with empty-plasmid-transfected RM-1 cells survived the experiment. As shown in Figure 4D, immunohistochemistry demonstrated that expression of VEGF became observably higher in the group receiving PSMA-transfected cells than in the group receiving empty-plasmid-transfected cells. The chi-square test $\left(X^{2}=9.926, P=0.002, P<0.05\right)$ specifically revealed strong positive VEGF staining ( $\geq 25 \%$ positive cells) in the group receiving PSMA-transfected cells $(16 / 19,84.21 \%)$ compared to the control group (6/18, 33.33\%).

\section{Infection with PSMA promotes the expression of MMP-9 in $\mathrm{PCa}$ osseous metastasis tissue}

Nineteen mice injected with PSMA-transfected RM-1 cells and 18 mice injected with empty-plasmid-transfected RM-1 cells survived the experiments. As shown in Figure $5 \mathrm{D}$, immunohistochemistry demonstrated that the expression of MMP-9 was noticeably higher in the group injected

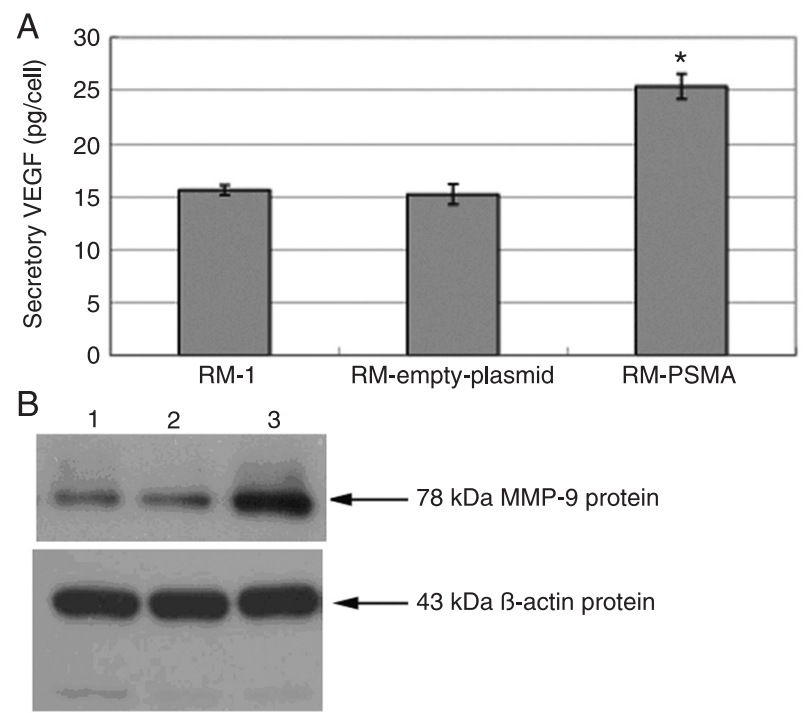

Figure 2. Expression of VEGF, MMP-9, and $\beta$-actin protein in cell lines. A, Quantification of VEGF protein by ELISA; ${ }^{*} \mathrm{P}<0.01$ for RM-PSMA cells compared to RM- 1 cells and negative control cells (one-way ANOVA). $B$, Western blot assay of MMP-9: lane 1 = MMP-9 protein in RM-1 cells; lane 2 = MMP-9 protein in empty-plasmid-transfected RM-1 cells; lane 3 = MMP-9 protein in PSMA-transfected RM-1 cells. VEGF = vascular endothelial growth factor; MMP-9 = matrix metalloproteinase-9; PSMA = prostate-specific membrane antigen.
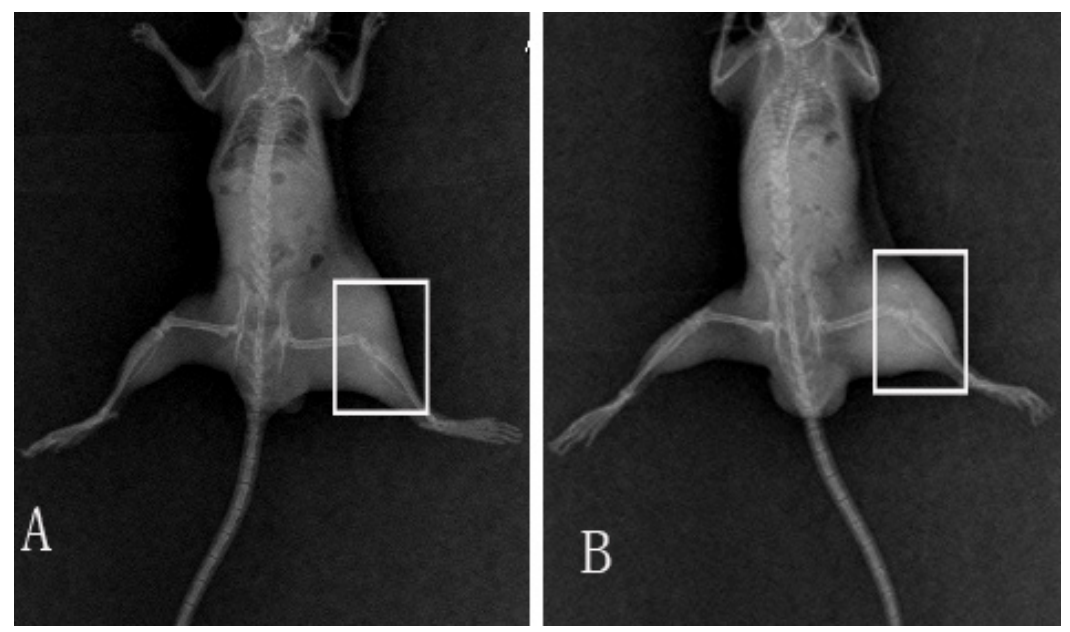

Figure 3. Radiologic detection and assays of femoral bone density. A, X-ray of an animal with prostate-specific membrane antigen-transfected RM-1 cells in the right femoral bone (osteolytic lesion in the area delimited by the box); $B, \mathrm{X}$-ray of an animal injected with empty-plasmid-transfected RM-1 in the right femoral bone (osteoblastic lesions in the area delimited by the box). with PSMA-transfected cells. As observed for VEGF, a strong positive expression of MMP-9 ( $\geq 25 \%$ positive cells) was detected in the group injected with PSMA-transfected cells $(14 / 19,73.68 \%)$, significantly higher than in the group injected with empty-plasmid-transfected cells (4/18, 
$22.22 \%)$, as shown by the chi-square test $\left(X^{2}=9.799\right.$, $P=0.002, P<0.05)$.

\section{Discussion}

In this study, radiographic imaging examination (bone density test) and pathologic examination were combined to demonstrate the successful induction of osseous metastasis in an animal model. In the animals injected with RM-1 cells, a reduction of osteolytic lesions and an increase of osteoblastic lesions were observed, with stable expression of PSMA. The bone density of the empty-transfected RM-1 cell group was higher than that of the RM-PSMA-transfected cell group. These findings support our hypothesis that the osteolytic function of PCa cell lines (RM-1) would be promoted by PSMA in the presence of in vivo osseous metastasis.

Prostate cancer commonly tends to produce bone metastases, which frequently lead to significant mutilation

Table 2. Sclerotic lesions and lytic lesions in osseous metastasis of prostate cancer.

\begin{tabular}{lcccc}
\hline Cell lines & Sclerotic lesions & Lytic lesions & Total & Lytic rate (\%) \\
\hline RM-empty plasmid & 13 & 5 & 18 & 27.8 \\
RM-PSMA & 6 & 13 & 19 & 68.4 \\
\hline
\end{tabular}

PSMA = prostate-specific membrane antigen.
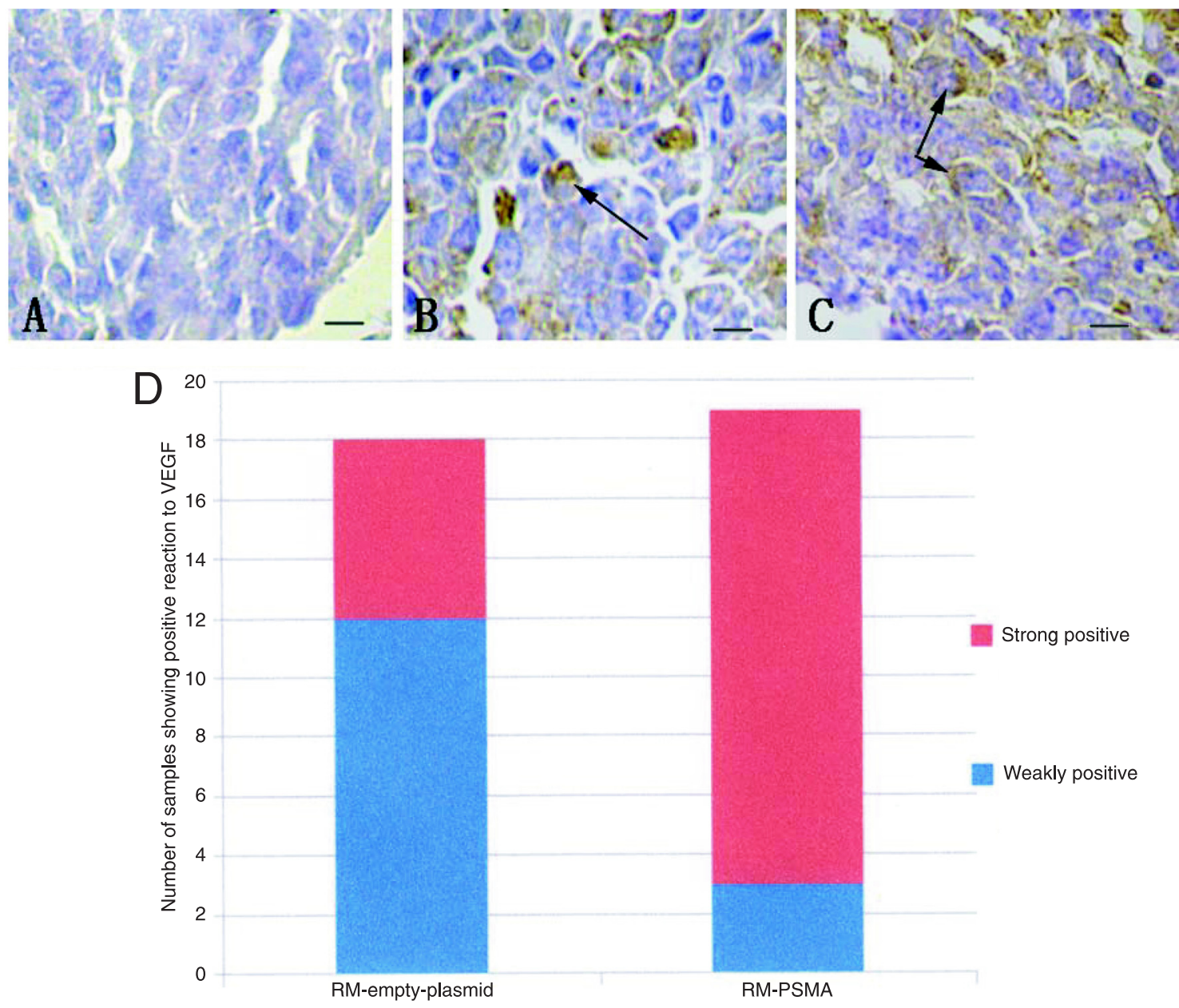

Figure 4. Immunohistochemistry of vascular endothelial growth factor (VEGF) in an osseous metastasis of prostate cancer. $A$, Negative (positive cells $\leq 5 \%$, scale bar $=25 \mu \mathrm{m}$ ); $B$, weakly positive (scale bar $=25 \mu \mathrm{m}$ ); arrows point to positive cells (positive expression of VEGF stained brown-yellow in cell membrane and cytoplasm); $C$, strongly positive (scale bar $=25 \mu \mathrm{m}$ ); arrows point to positive cells (positive expression of VEGF stained brown-yellow in cell membrane and cytoplasm); $D$, number of samples showing positive reaction to VEGF. PSMA = prostate-specific membrane antigen. 

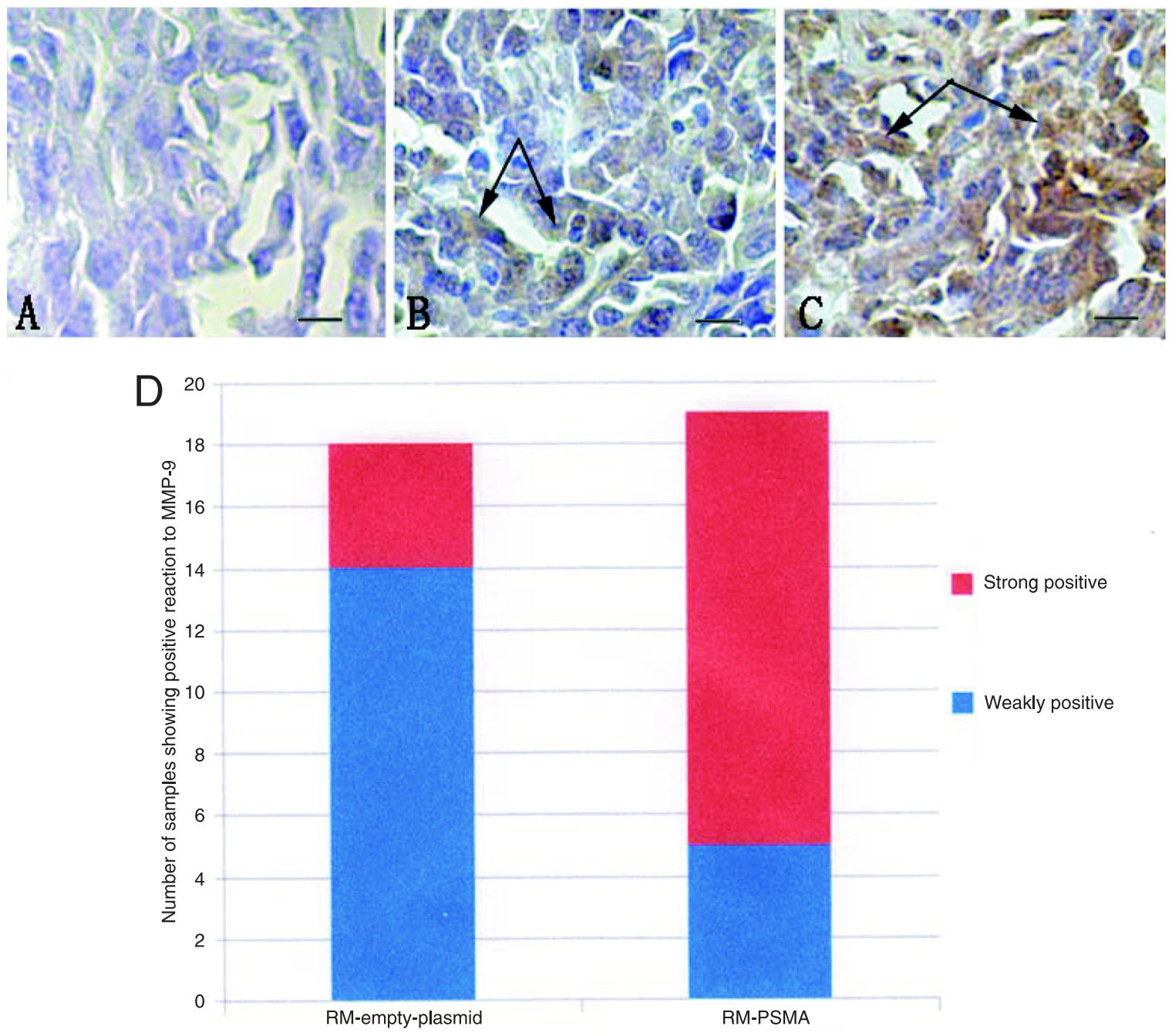

Figure 5. Immunohistochemistry of matrix metalloproteinase-9 (MMP-9) in an osseous metastasis of prostate cancer. $A$, Negative (positive cells $\leq 5 \%$, scale bar $=25 \mu \mathrm{m}$ ); $B$, weakly positive (scale bar $=25 \mu \mathrm{m}$ ); arrows point to positive cells (positive expression of MMP-9 stained brown-yellow in cell membrane and cytoplasm); $C$, strongly positive (scale bar $=25 \mu \mathrm{m}$ ); arrows point to positive cells; $D$, number of samples showing positive reaction to MMP-9 (positive expression of MMP-9 stained brown-yellow in cell membrane and cytoplasm). PSMA = prostate-specific membrane antigen.

and mortality. It is generally accepted that PCa osseous metastases are predominantly osteoblastic but with underlying osteolytic lesions. Histomorphologic studies and radiographs have confirmed that both bone formation and bone breakdown are present within PCa osseous metastases $(22,23)$.

Osteoblastic lesions generally predominate in the earlier phase, but the occurrence and increase of osteoblastic lesions have been clinically observed in PCa patients with osseous metastases with the aggravation of the disease. Some earlier studies even reported a marked increase of PSMA expression in PCa recurrence and metastasis $(4,21)$.
This PCa process was simulated and confirmed in our study. With the methods described above, animal models of PCa osseous metastasis were constructed, providing a new platform for $\mathrm{PCa}$-relevant research. We assumed that the rate of hormone-resistant PCa cells, highly PSMA expressing, would increase in patients with recurrent and metastatic PCa. The occurrence and increase of osteolytic lesions could then be induced since in vivo bone invasion and breakdown were higher than the proliferation and calcification of these PCa cells. It is not merely hypothetical that animal models could be consistent with clinic processes in $\mathrm{PCa}$ diagnosis and treatment.

As mentioned above, the adhesion, migration and 
invasion of PCa cell lines are definitely important factors influencing $\mathrm{PCa}$ osseous metastases. Some recent reports also focused on the influence of PSMA on the in vitro biological function of PCa cell lines. Barwe et al. (12), Ghosh and Heston (15), and Rajasekaran et al. (13) suggested that PSMA promote in vitro adhesion and migration of PCa cell lines. However, few studies have examined and delineated regulation and/or mechanism involved.

The development of a neovascular network, also known as angiogenesis, has been proved to be important in the development and metastasis of a variety of human solid tumors $(24,25)$. Tumor cells can secrete a variety of angiogenesis factors that promote neovascularization. Angiogenesis factors thus probably control neovascularization by regulating the proliferation and migration of vascular endothelial cells, both of them critical steps in tumor neovascularization. In 1993, Stetler-Stevenson et al. (26) reported a positive correlation between increasing levels of VEGF and tumor metastasis, which now has been generally accepted as the strongest angiogenesis factor. Some other investigations have demonstrated the abilities of invasion and metastasis of a variety of PCa cell lines (27). These studies have shown that VEGF, a growth factor autocrined by tumor cells, can promote division growth and inhibit apoptosis of tumor cells $(28,29)$.

The present study proposed and confirmed that PSMA has a significant correlation with VEGF expression in the RM-1 PCa cell line, in agreement with reports by Ochida et al. (30) and Chen et al. (31). Chen et al. (31) reported that the level of VEGF expression is positively correlated with Glean score and proposed that the pathologic grade of PCa and VEGF could promote the migration of PCa cells in osseous metastases, which appear to have a higher expression of VEGF than PCa in situ (also in 24,32,33). Our results, based on immunohistochemical analyses of animal models and on ELISA, support the findings of the above studies, proposing that PSMA promotes the migration of the RM-1 PCa cell line in vivo by increasing the expression of VEGF.

With respect to MMPs, our results regarding both in vitro $\mathrm{RM}-1$ cells and in vivo PCa osseous metastases in a mouse model indicate that the expression of MMP-9 in RM-1 cell lines was enhanced by PSMA. They may also suggest that the degradation of the basement membrane and extracellular

\section{References}

1. Emonds KM, Swinnen JV, Mortelmans L, Mottaghy FM. Molecular imaging of prostate cancer. Methods 2009; 48: 193-199.

2. Mhawech-Fauceglia P, Zhang S, Terracciano L, Sauter G, Chadhuri A, Herrmann FR, et al. Prostate-specific membrane antigen (PSMA) protein expression in normal and neoplastic tissues and its sensitivity and specificity in prostate adenocarcinoma: an immunohistochemical study using mutiple tumour tissue microarray technique. Histopathology 2007; 50: 472-483. matrix of RM-1 cells is enhanced with increasing expression of MMP-9, effectively promoted by PMSA-transfected cells. The result might be a promotion of both the invasion and migration abilities of the RM-1 cell line.

In fact, MMPs play a series of roles in both the invasion and metastasis of PCa cells (34). Secreted by invasive tumor cells, MMPs can degrade the basement membrane and extracellular matrix, which represent the major defensive barrier. More specifically, participating in the degradation of the major component, type $\chi$ collagen, MMP-9 plays a critical role in angiogenesis, invasion, and formation of metastasis in a variety of cancers (35). The enhanced expression and/ or activity of MMP-9 in the epithelium of malignant tumors has been widely accepted (36). According to some investigations, the invasiveness and bone resorption ability of the PCa cell line PC-3 was specifically facilitated by up-regulated expression of MMP-9 (37). However, a limited number of studies have been conducted on the relationship between MMP-9 and PSMA in PCa osseous metastases in vivo.

To sum up, although further studies are needed about how PSMA exactly promotes the expression of both VEGF and MMP-9, on the basis of the present study, we conclude that: 1) PSMA can increase the expression of VEGF and MMP-9 in PCa cells both in vitro and in vivo, thus promoting tumor invasion and destruction of osseous tissue; 2) by increasing sclerotic destruction of PCa cells, PSMA can promote in vivo osseous metastasis of $\mathrm{PCa}$, and 3 ) as a result, the occurrence and increase of osteolytic lesions were induced, as in vivo bone invasion and breakdown were higher than proliferation and calcification of these $\mathrm{PCa}$ cells, which highly expressed PSMA. Then, PCa osseous metastasis progressed to an advanced stage.

The present study also indicates that PSMA should become an attractive target for treatment of osseous metastases of PCa.

\section{Acknowledgments}

Research supported by the National Natural Science Foundation (\#3087584, \#81001146), the Guangdong Natural Science Foundation (\#8251008901000018 and \#9451008901002062), and the Fund for the Preceptorial Program of Higher Education (\#20090171120062).
3. Marchal C, Redondo M, Padilla M, Caballero J, Rodrigo I, Garcia J, et al. Expression of prostate specific membrane antigen (PSMA) in prostatic adenocarcinoma and prostatic intraepithelial neoplasia. Histol Histopathol 2004; 19: 715-718.

4. Dorer DE, Nettelbeck DM. Targeting cancer by transcriptional control in cancer gene therapy and viral oncolysis. Adv Drug Deliv Rev 2009; 61: 554-571.

5. Zhang L, Wang CY, Yang R, Shi J, Fu R, Chen L, et al. Real- 
time quantitative RT-PCR assay of prostate-specific antigen and prostate-specific membrane antigen in peripheral blood for detection of prostate cancer micrometastasis. Urol Oncol 2008; 26: 634-640.

6. Ikegami S, Tadakuma T, Ono T, Suzuki S, Yoshimura I, Asano $\mathrm{T}$, et al. Treatment efficiency of a suicide gene therapy using prostate-specific membrane antigen promoter/enhancer in a castrated mouse model of prostate cancer. Cancer Sci 2004; 95: 367-370.

7. Wang K, Gao X, Pang J, Liu X, Cai Y, Zhang Y, et al. Dendritic cells transduced with a PSMA-encoding adenovirus and cocultured with autologous cytokine-induced lymphocytes induce a specific and strong immune response against prostate cancer cells. Urol Oncol 2009; 27: 26-32.

8. Zhao W, Xu Y, Kong D, Liu R, Zhang Z, Jin C, et al. Tissueselective RNA interference in prostate cancer cell using prostate specific membrane antigen promoter/enhancer. Urol Oncol 2009; 27: 539-547.

9. Conway RE, Petrovic N, Li Z, Heston W, Wu D, Shapiro LH. Prostate-specific membrane antigen regulates angiogenesis by modulating integrin signal transduction. Mol Cell Biol 2006; 26: $5310-5324$

10. Wang W, Tavora F, Sharma R, Eisenberger M, Netto GJ. PSMA expression in Schwannoma: a potential clinical mimicker of metastatic prostate carcinoma. Urol Oncol 2009; 27: 525-528.

11. Voorzanger-Rousselot N, Garnero P. Biochemical markers in oncology. Part I: molecular basis. Part II: clinical uses. Cancer Treat Rev 2007; 33: 230-283.

12. Barwe SP, Maul RS, Christiansen JJ, Anilkumar G, Cooper CR, Kohn DB, et al. Preferential association of prostate cancer cells expressing prostate specific membrane antigen to bone marrow matrix. Int J Oncol 2007; 30: 899-904.

13. Rajasekaran AK, Anilkumar G, Christiansen JJ. Is prostatespecific membrane antigen a multifunctional protein? $A m \mathrm{~J}$ Physiol Cell Physiol 2005; 288: C975-C981.

14. Haffner MC, Kronberger IE, Ross JS, Sheehan CE, Zitt M, Muhlmann $\mathrm{G}$, et al. Prostate-specific membrane antigen expression in the neovasculature of gastric and colorectal cancers. Hum Pathol 2009; 40: 1754-1761.

15. Ghosh A, Heston WD. Tumor target prostate specific membrane antigen (PSMA) and its regulation in prostate cancer. $J$ Cell Biochem 2004; 91: 528-539.

16. Ghosh A, Wang X, Klein E, Heston WD. Novel role of prostatespecific membrane antigen in suppressing prostate cancer invasiveness. Cancer Res 2005; 65: 727-731.

17. Andela VB, Gordon AH, Zotalis G, Rosier RN, Goater JJ, Lewis $\mathrm{GD}$, et al. NFkappaB: a pivotal transcription factor in prostate cancer metastasis to bone. Clin Orthop Relat Res 2003; S75S85.

18. Cao KY, Mao XP, Wang DH, Xu L, Yuan GQ, Dai SQ, et al. High expression of PSM-E correlated with tumor grade in prostate cancer: a new alternatively spliced variant of prostate-specific membrane antigen. Prostate 2007; 67: 1791-1800.

19. Sacha P, Zamecnik J, Barinka C, Hlouchova K, Vicha A, Mlcochova $\mathrm{P}$, et al. Expression of glutamate carboxypeptidase II in human brain. Neuroscience 2007; 144: 1361-1372.

20. Thomas AG, Wozniak KM, Tsukamoto T, Calvin D, Wu Y, Rojas C, et al. Glutamate carboxypeptidase II (NAALADase) inhibition as a novel therapeutic strategy. Adv Exp Med Biol 2006; 576: 327-337.

21. Denmeade SR, Sokoll LJ, Dalrymple S, Rosen DM, Gady AM,
Bruzek D, et al. Dissociation between androgen responsiveness for malignant growth vs. expression of prostate specific differentiation markers PSA, hK2, and PSMA in human prostate cancer models. Prostate 2003; 54: 249-257.

22. Reichert JC, Quent VM, Burke LJ, Stansfield SH, Clements JA, Hutmacher DW. Mineralized human primary osteoblast matrices as a model system to analyse interactions of prostate cancer cells with the bone microenvironment. Biomaterials 2010; 31: 7928-7936.

23. Akimoto $\mathrm{S}$, Furuya $\mathrm{Y}, \mathrm{Akakura} \mathrm{K}$, Ito $\mathrm{H}$. Comparison of markers of bone formation and resorption in prostate cancer patients to predict bone metastasis. Endocr J 1998; 45: 97-104.

24. Jennbacken K, Vallbo C, Wang W, Damber JE. Expression of vascular endothelial growth factor C (VEGF-C) and VEGF receptor-3 in human prostate cancer is associated with regional lymph node metastasis. Prostate 2005; 65: 110-116.

25. Skobe M, Hawighorst T, Jackson DG, Prevo R, Janes L, Velasco P, et al. Induction of tumor lymphangiogenesis by VEGF-C promotes breast cancer metastasis. Nat Med 2001; 7: 192-198.

26. Stetler-Stevenson WG, Aznavoorian S, Liotta LA. Tumor cell interactions with the extracellular matrix during invasion and metastasis. Annu Rev Cell Biol 1993; 9: 541-573.

27. Qi L, Robinson WA, Brady BM, Glode LM. Migration and invasion of human prostate cancer cells is related to expression of VEGF and its receptors. Anticancer Res 2003; 23: 3917-3922.

28. Masood R, Cai J, Zheng T, Smith DL, Hinton DR, Gill PS. Vascular endothelial growth factor (VEGF) is an autocrine growth factor for VEGF receptor-positive human tumors. Blood 2001; 98: 1904-1913.

29. Ehrenfried JA, Ko TC, Thompson EA, Evers BM. Cell cyclemediated regulation of hepatic regeneration. Surgery 1997; 122 : 927-935.

30. Uchida A, O'Keefe DS, Bacich DJ, Molloy PL, Heston WD. In vivo suicide gene therapy model using a newly discovered prostate-specific membrane antigen promoter/enhancer: a potential alternative approach to androgen deprivation therapy. Urology 2001; 58: 132-139.

31. Chen J, De S, Brainard J, Byzova TV. Metastatic properties of prostate cancer cells are controlled by VEGF. Cell Commun Adhes 2004; 11: 1-11.

32. Walsh K, Sriprasad S, Hopster D, Codd J, Mulvin D. Distribution of vascular endothelial growth factor (VEGF) in prostate disease. Prostate Cancer Prostatic Dis 2002; 5: 119-122.

33. Mann R, Mulligan RC, Baltimore D. Construction of a retrovirus packaging mutant and its use to produce helper-free defective retrovirus. Cell 1983; 33: 153-159.

34. Miller $A D$, Buttimore $C$. Redesign of retrovirus packaging cell lines to avoid recombination leading to helper virus production. Mol Cell Biol 1986; 6: 2895-2902.

35. Britton KE, Feneley MR, Jan H, Chengazi VU, Granowska M. Prostate cancer: the contribution of nuclear medicine. BJU Int 2000; 86 (Suppl 1): 135-142.

36. Davies B, Waxman J, Wasan H, Abel P, Williams G, Krausz $\mathrm{T}$, et al. Levels of matrix metalloproteases in bladder cancer correlate with tumor grade and invasion. Cancer Res 1993; 53: 5365-5369.

37. Sehgal G, Hua J, Bernhard EJ, Sehgal I, Thompson TC, Muschel RJ. Requirement for matrix metalloproteinase-9 (gelatinase B) expression in metastasis by murine prostate carcinoma. Am J Pathol 1998; 152: 591-596. 